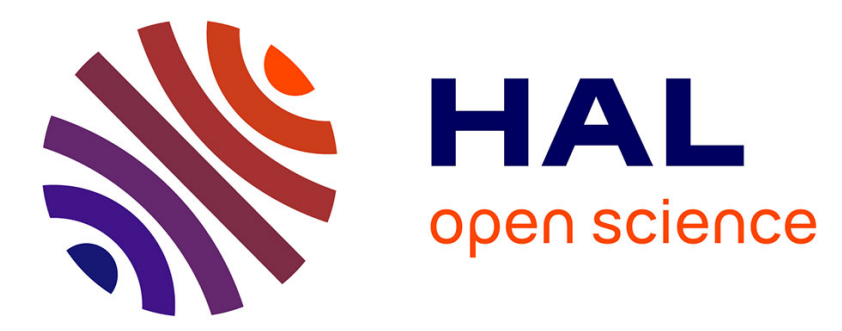

\title{
Weighing the importance of model uncertainty against parameter uncertainty in earthquake loss assessments
}

Jeremy Rohmer, John Douglas, Didier Bertil, Daniel Monfort, Olivier Sedan

\section{To cite this version:}

Jeremy Rohmer, John Douglas, Didier Bertil, Daniel Monfort, Olivier Sedan. Weighing the importance of model uncertainty against parameter uncertainty in earthquake loss assessments. Soil Dynamics and Earthquake Engineering, 2014, 58, pp.1-9. 10.1016/j.soildyn.2013.11.006 . hal-00924122

\section{HAL Id: hal-00924122 \\ https://hal-brgm.archives-ouvertes.fr/hal-00924122}

Submitted on 6 Jan 2014

HAL is a multi-disciplinary open access archive for the deposit and dissemination of scientific research documents, whether they are published or not. The documents may come from teaching and research institutions in France or abroad, or from public or private research centers.
L'archive ouverte pluridisciplinaire HAL, est destinée au dépôt et à la diffusion de documents scientifiques de niveau recherche, publiés ou non, émanant des établissements d'enseignement et de recherche français ou étrangers, des laboratoires publics ou privés. 
1 Weighing the importance of model uncertainty against

2 parameter uncertainty in earthquake loss assessments

4 Jeremy Rohmer ${ }^{a 1}$, John Douglas ${ }^{a}$, Didier Bertil $^{a}$, Daniel Monfort ${ }^{a}$, Olivier Sedan ${ }^{a}$

$5 \quad$ aaRGM, 3 av. C. Guillemin, B.P. 36009, 45060 Orléans Cédex 2, France

6

7 Abstract

8 Epistemic uncertainties can be classified into two major categories: parameter and model.

9 While the first one stems from the difficulties in estimating the values of input model parameters, the second comes from the difficulties in selecting the appropriate type of model.

11 Investigating their combined effects and ranking each of them in terms of their influence on 12 the predicted losses can be useful in guiding future investigations. In this context, we propose 13 a strategy relying on variance-based global sensitivity analysis, which is demonstrated using 14 an earthquake loss assessment for Pointe-à-Pitre (Guadeloupe, France). For the considered 15 assumptions, we show: that uncertainty of losses would be greatly reduced if all the models could be unambiguously selected; and that the most influential source of uncertainty (whether of parameter or model type) corresponds to the seismic activity group. Finally, a sampling strategy was proposed to test the influence of the experts' weights on models and on the assumed coefficients of variation of parameter uncertainty. The former influenced the sensitivity measures of the model uncertainties, whereas the latter could completely change the importance rank of the uncertainties associated to the vulnerability assessment step.

Keywords: seismic risk; economic direct loss; epistemic uncertainty; variance-based global sensitivity analysis; Sobol' indices; ranking

${ }^{1}$ Corresponding author: Jeremy Rohmer, j.rohmer@brgm.fr

Tel: (+33) (0)2 386430 92, Fax: (+33) (0)2 38643689 


\section{Introduction}

2 Predicting the consequences (losses) of future earthquakes is of primary importance for risk management. One of the greatest challenges when building such predictive models is the treatment of the multiple sources of uncertainty pervading the different steps of earthquake loss assessment (seismic source, wave propagation, local site effects, exposed inventory, vulnerability assessment, and damage and loss estimation). This issue has been highlighted by numerous authors over the last decades [e.g., 1, 2, 3, 4, 5]. It is now common to classify uncertainty into randomness (aleatory variability) and knowledge-based uncertainty (epistemic uncertainty) [e.g., 6]. Though appearing to be partly a choice of the modeller [7], this categorization can be useful in practice, because decisions motivated by the aleatory variability will be quite different from decisions based on the knowledge-based uncertainty. In the first case, no concrete actions can reduce randomness and only "indirect" actions can be proposed by means of protective or preventive measures. In the second, concrete actions can be undertaken to act directly on the uncertainty reduction and the best solution is to set priorities for data collection/analysis under budget constraints on the basis of the identification of the most influential sources of knowledge-based uncertainties [e.g., 8].

To reduce epistemic uncertainties, sensitivity analysis can provide valuable information by addressing the following questions: what sources of uncertainty contribute the most to the uncertainties in the predicted losses? And at what stages of the loss assessment procedure (e.g., hazard, vulnerability or damage evaluation)? How to rank these sources of uncertainties? And how to set priorities for future investigations? The great value of addressing such questions has long been recognized in the field of seismic risk assessments and, more specifically, for probabilistic seismic hazard assessment (PSHA) relying on logic trees [9]. Two main approaches have been followed: a "one-factor-at-a-time" (OAT) approach analysing variations from a base model results by varying, in turn, the input parameters or considering different scenarios [3, 10]; and the multi-parameter method based on factorial designs allowing simultaneous changes to parameters on the branches of a logic tree [11]. This approach was applied in an Italian case study by Barani et al. [12].

In the present article, we focus on two types of epistemic uncertainty, which are those most commonly encountered in practice: parameter and model. The first category stems from the difficulties in estimating the input parameters (in a broad sense) of models/analysis due to the limited number, poor representativeness (caused by time, space and financial limitations), and 
1 imprecision of observations/data. In addition, models are necessarily simplified

2 representations of the phenomena, i.e. they are based on assumptions, and compliance

3 between the model assumptions and the properties of the system being analysed never exist in

4 an absolute sense [e.g., 13]. Uncertainty can then appear in the structure/form of the model, which depends on the choice of variables, dependencies, processes and so forth regarded as relevant and prominent for their purpose in the model. Yet, in some cases, a set of different models (e.g. differing in their structure and input variables) are either considered equally adequate (e.g., they equally fit the observations), or they are associated with different confidence levels. This is exemplified by the extensively debated issue of selecting appropriate ground motion prediction equations (GMPE, [e.g., 14]).

11 In this context, the objective of the present article is to show how recent advances in global sensitivity analysis [15] can provide valuable information to answer the following questions for earthquake loss estimation: What is the contribution of model uncertainty to the uncertainty in predicted losses when simultaneously accounting for parameter uncertainty? How to measure such a contribution? Should future investigations spend effort on the modelling procedure or on parameter estimation? In this view, we propose a strategy based on variance-based sensitivity analysis (VBSA), which can overcome some limitations of the afore-mentioned sensitivity approaches (as discussed in Section 2).

The remainder of the present paper is organized as follows. In the next section, we describe the global sensitivity analysis using VBSA and the method for both investigating sensitivity to model and parameter uncertainty. Such a strategy is applied to predictions of direct monetary losses for the city of Pointe-à-Pitre (Guadeloupe, France) using simplified information on uncertainty (but based on real data). The following section describes the earthquake loss model and the case study of Pointe-à-Pitre (context and assumptions for representing the different uncertainty sources). It should be underlined that the application to Pointe-à-Pitre has been chosen for demonstration purposes only and all the presented results should not be interpreted as a definitive uncertainty assessment. The subsequent section shows the results of the VBSA and discusses how results are modified by changing the size of the different sources of uncertainty. The article ends with some brief conclusions and suggestions for future work. 


\section{Investigating sensitivity to model and parameter uncertainty}

2 In this section we present the technique used here to assess the sensitivity of the results to the

3 two different types of uncertainty.

\section{$4 \quad 2.1 \quad$ Variance-based sensitivity analysis}

5 VBSA is a stochastic method providing a quantitative measure of sensitivity $[16,17]$ assigned

6 to each source of uncertainty (represented by any kind of probabilistic distribution, e.g. uniform, normal or discrete). VBSA presents the advantages of exploring the sensitivity over the whole range of variation (i.e. in a global manner) of the input random variables and of fully accounting for possible interactions between them. This is contrary to the OAT technique, as discussed by Saltelli and Annoni [18]. VBSA allows identification of:

- which input parameters contribute the most to the output variability (within the "factors' prioritisation setting" as described by Saltelli et al. [15]) through the use of the Sobol' indices of first order, also called main effects (see below for a formal description);

- which input parameters are insignificant and can be eliminated to "simplify" the model (within a "factors' fixing setting" as described by Saltelli et al. [15]) through the use of total effects (see below for a formal description).

By comparing the main and total effects, this technique improves insight into the nature of the considered model. For instance, the case where main and total effects are of equal importance and the sum of the main effects nearly reach unity, indicates that the uncertainty of the output (i.e. the variance) is only due to the sum of the effects of each uncertain parameter taken alone, and not from interactions among them. Thus, the model can be simplified and be represented as a sum of elementary one-dimensional functions of the input parameters. Formally, the model is said to be "additive". Conversely, if the main effects have low values compared to the total effects, this indicates strong high-order interactions between the parameters, hence a model of high complexity. Thus, VBSA helps to explore the model behaviour in the domain of variation of the input parameters, which can be of great value when using a loss model in a black-box fashion (see discussion provided by Bommer et al. 
1 [4]). More recently, the overview of the model complexity brought by VBSA has been better

2 formalized with the notion of effective dimension [19], which can be understood as the 3 number of dominant parameters of the model.

4 Finally, VBSA is general in the sense that it applicable to any kind of model (linear, non5 linear, additive and so forth), i.e. without introducing a priori assumptions on its mathematical structure [15]. For instance, simultaneously varying the extreme values of parameters (e.g. using a two-level factorial design as carried out by Rabinowitz and Steinberg [11]) only shows good results for quasi-linear models.

\section{$2.2 \quad$ Brief mathematical description}

We introduce here the basic concepts of VBSA. For a more complete introduction and full derivation of the equations, the interested reader is referred to Saltelli et al. [15] and references therein.

Let us define $f$ as the earthquake loss model. Considering the $n$-dimensional vector $\boldsymbol{X}$ as a random vector of independent random variables $X_{i}(\mathrm{i}=1,2, \ldots, n)$, then the output $Y=f(\boldsymbol{X})$ is also a random variable (as a function of a random vector). VBSA aims to determine the part of the total unconditional variance $\operatorname{Var}(Y)$ of the output $Y$ resulting from each input random variable $X_{i}$

In practice, the partial and total variances of $Y$ are determined based on the decomposition of $f$ (i.e. functional analysis of variance decomposition of $f$ as proposed by Sobol' [16]), into summands of increasing dimension (provided that $f$ can be integrated). Each of these terms can be evaluated through multidimensional integrals, which can be approximated through Monte-Carlo-based algorithms (see [20], for a recent review and discussion). For instance, the sequential algorithm of Saltelli et al. [20], using a formula of Jansen [21], requires a total of $N(n+2)$ model evaluations, where $N$ is the number of Monte-Carlo samples and $n$ is the number of input uncertain parameters $X_{\mathrm{i}}$. This is noteworthy as the quality of the MonteCarlo-based approach directly depends on the sample size $N$. The interested reader is referred to Saltelli et al. [15] for a review of other computational procedures.

28 On this basis, the Sobol' indices (ranging between null and unity) can be defined as:

$$
S_{i}=\frac{\operatorname{Var}\left[\mathrm{E}\left(Y \mid X_{i}\right)\right]}{\operatorname{Var}(Y)}, \quad S_{i j}=\frac{\operatorname{Var}\left[\mathrm{E}\left(Y \mid X_{i}, X_{j}\right)\right]}{\operatorname{Var}(Y)}-S_{i}-S_{j}
$$


1 The first-order $S_{\mathrm{i}}$ is referred to as "the main effect of $X_{i}$ " and can be interpreted as the

2 expected proportion of the total variance of the output $Y$ (i.e. representing the uncertainty in

$3 \quad Y$ ) that would be removed if we were able to learn the true value of $X_{\mathrm{i}}$. This index provides a

4 measure of importance (i.e. a sensitivity measure) useful when ranking, in terms of

5 importance, the different input parameters [15]. The second order term $S_{\mathrm{ij}}$ measures the combined effect of both parameters $X_{i}$ and $X_{j}$. Higher order terms can be defined in a similar fashion. The total number of sensitivity indices reaches $2^{n}-1$. Sensitivity analysis is generally limited, however, to the pair of indicators corresponding to the main effect $S_{i}$ and to the total effect $S_{T i}$ of $X_{\mathrm{i}}$ (see e.g., [15]). The latter is defined as follows:

$S_{T i}=1-\frac{\operatorname{Var}\left[\mathrm{E}\left(Y \mid X_{-i}\right)\right]}{\operatorname{Var}(Y)}$

where $X_{-i}=\left\{X_{1}, \ldots, X_{i-1}, X_{i+1}, \ldots X_{n}\right\}$. The total index corresponds to the fraction of the uncertainty in $Y$ that can be attributed to $X_{\mathrm{i}}$ and its interactions with all other input parameters. $S_{T i}=0$ implies that the input factor $X_{\mathrm{i}}$ has no effect and so $X_{\mathrm{i}}$ can be fixed at any value over its uncertainty range (factors' fixing setting).

As afore-mentioned, the relationship between the main and total effects helps to explore the model complexity. Several cases have been formalized by Kucherenko et al. [19]. In particular, if $S_{\mathrm{i}} \approx S_{\mathrm{j}}$ for any $\mathrm{i}$ and $\mathrm{j}$ and $\mathrm{S}_{\mathrm{i}} \approx S_{T i}$ then the model has equally important variables, but with "weak" interaction among them. Conversely, if $S_{\mathrm{i}} \approx S_{\mathrm{j}}$ for any $\mathrm{i}$ and $\mathrm{j}$ and $S_{\mathrm{i}}<<S_{T i}$ then the model has equally important variables, but with "strong" interaction among them.

\section{$2.3 \quad$ Method}

The method adopted here relies on the main and total effects. Parameter uncertainties are classically assigned a probability distribution (e.g., uniform or normal) according to which the samples are generated to estimate the main effects. In practice, the number of parameters of loss assessment models can be large (>20) and we advocate using a Monte-Carlo strategy relying on Sobol' quasi-random sequences (e.g., LP- $\tau$ sequence) allowing the generation of samples as uniformly as possible (see e.g., [20]). This can outperform crude Monte-Carlo sampling in the estimation of multi-dimensional integrals [17]. Besides, instead of calculating the pair of sensitivity measures for each model parameter, we propose to define such sensitivity indices for "groups of variables". The definition provided by Eq. 1 can be 
generalized to a set of random variables (see the formal definition and discussion in Saltelli et al. [15], section 1.2.15). Examples of such groups for the example shown here are the groups of: seismic activity parameters, vulnerability indices and site amplification factors (see

4 Section 3.3).

Model uncertainties can be treated by assigning to a family of plausible models, a discrete random variable, i.e. randomly taking its value from a set of finite values. For instance, when four different models are considered plausible, the discrete variable takes its value from the set $\{1,2,3,4\}$, each integer value corresponding to a given model. If all the different models are considered equally adequate, i.e. experts' weights of confidence are equal (to $25 \%$ in this example); then the discrete random variable is uniformly generated. Conversely, if the confidence weights are different, they can be used to constrain the random generation.

\section{Example application}

In this section, we present: the context of the Pointe-à-Pitre application (Section 3.1), the earthquake loss model applied to predict direct monetary losses associated to future earthquake events (Section 3.2), and the assumptions made to represent both model and parameter uncertainty of the loss model (Sections 3.3 and 3.4).

\subsection{Pointe-à-Pitre's context}

The study is focused on the Pointe-à-Pitre urban area, the main city of Guadeloupe, an island in the French Antilles (see location in Fig. 1A), which represents more than half the island's population. Guadeloupe is situated in the Lesser Antilles arc, which is a zone of convergence between the South American and the Caribbean plates at a rate of about $2 \mathrm{~cm} / \mathrm{yr}$ [22]. This movement is absorbed by the subduction of the South American plate below the Caribbean plate and deformation of the wedge of the upper plate on a 100-250 km-wide zone, producing an extended system of active crustal faults. It results in a high seismicity level (about 1,000 detected events per year [23]), making this region particularly exposed to seismic risk, as demonstrated by major earthquakes in 1843 and 1897 (see http://sisfrance.brgm.fr/Antilles/), which were particularly devastating for Pointe-à-Pitre. 


\subsection{Earthquake loss assessment model}

2 Hereafter, we describe the earthquake loss assessment model used, which is mainly based on 3 the procedure described by Sedan et al. [24] and references therein. It should be underlined 4 that the objective of the present article is not the development of a sophisticated earthquake loss model, but the application of a method to investigate the sensitivity to model and parameter uncertainty. This approach could be applied to any kind of loss assessment. Examples of more advanced earthquake loss assessment procedures are provided by Crowley et al. [3], for instance.

The evaluation is restricted to the tangible economic losses to residential dwellings, i.e. to direct losses associated with physical damage to buildings related both to the repair of structural damage and the replacement of building contents. The costs associated to damage to equipment within buildings are not considered.

The procedure is comprised of three main steps. The first one aims to estimate the local hazard. A single ground-motion parameter/intensity measure (peak ground acceleration, PGA) is chosen for this assessment. Starting from the PGA on bedrock derived from regional probabilistic hazard assessment, lithological site effects, which can cause significant amplification of earthquake ground motions, are accounted for by a constant multiplicative factor applied to the PGA on bedrock at a given location. Such amplification factors are estimated based a zonation of the studied region into zones, where the amplification is assumed to be spatially homogeneous. These zones are defined by the processing of different information (seismic microzonation): geological, geophysical (spectral analysis of surface waves and analysis of ambient vibrations) and geotechnical data. Pointe-à-Pitre is particularly prone to such site effects, as demonstrated by the 1985 Montserrat earthquake when Pointe-àPitre suffered more structural damage than other cities on Guadeloupe [25, 26]. These effects are mainly due to the particular geology of the area, characterized by the presence of small limestone hills and mangrove swamps filled with limestone from the surrounding hills.

The second step aims to estimate the physical damage to buildings using the European Macroseismic Scale (EMS-98) damage grades [27]. Six damage grades $D_{k}$ (with $k$ from 0 to 5) are considered: $\mathrm{D}_{0}=0$ corresponds to "no damage", $\mathrm{D}_{1}=1$ to "slight damage", $\mathrm{D}_{2}=2$ to " "moderate damage", $\mathrm{D}_{3}$ to "heavy damage", $\mathrm{D}_{4}=4$ to "very heavy damage" and $\mathrm{D}_{5}=5$ to "maximum damage', This assessment is mainly based on the macroseismic approach developed in the project Risk-UE, funded by the European Commission's $5^{\text {th }}$ Framework 
1 Programme [28]. This approach uses the macroseismic intensity defined according to EMS98. This is calculated by converting the local PGA derived from the hazard assessment using empirical equations. In the present study, a single conversion model, based on a combination of [29], for low intensities, and [30], for high intensities, was considered relevant. In the RiskUE method, the seismic behaviour of buildings is subdivided into vulnerability classes grouping different types of buildings that may perform in a similar way during earthquakes. The vulnerability is evaluated by means of the Vulnerability Index (VI) ranging from null to unity (i.e. least to most vulnerable). The studied urbanized area is subdivided into districts, which define sets of buildings (Figure 1B). Within each defined district, the ratio of vulnerability classes is evaluated by compiling an inventory based on either aerial photos or, more recently, satellite remote sensing and ground-based omnidirectional imaging [31] or site surveys (i.e. through visual inspection of a limited number of buildings chosen within the district). The combination of the macroseismic intensity, the vulnerability indices assigned to each typology and the distribution of vulnerability classes in each district allows computation of the distribution of the number of building per district in the six damage grades (see [28] for further details).

In the final step, the economic losses are quantified by converting the damage grades into monetary losses. This is conducted using empirical relationships linking the damage grade to the repair damage ratios (see, for instance, Table 2 in [32]), which represent the cost of repair divided by the cost of full replacement of the structure (assumed to be the construction cost). Based on the distribution of buildings per district in each damage grade, the repair damage ratios and the average replacement cost of each typology of buildings, the overall economic losses can be calculated by summing the contributions of the different districts.

\subsection{Sources of parameter uncertainty}

Within each phase of the afore-described loss-assessment model, different sources of parameter uncertainty can be identified (Table 1, groups "HAZ", "LITH", "VI" and "COST"). Below, we describe the assumptions made for their mathematical representations. The influence of these assumptions on the results of the sensitivity analysis (more particularly of the assumed coefficients of variation) is discussed in Section 4.3.

In the hazard assessment step, the first group of parameter uncertainties correspond to the seismicity activity for the PSHA, namely: the maximum magnitude; the annual occurrence 
rate for an anchoring magnitude; the focal depth and the b-value of the Gutenberg-Richter relation. The regional probabilistic hazard assessment used here (and also the basis of the Eurocode 8 seismic design map for this region, http://www.planseisme.fr/Zonage-sismiquede-la-France.html) was carried out in 2002 by Martin et al. [33] for the French Antilles (Guadeloupe and Martinique and other islands), with a spatial resolution of $0.1^{\circ}(\sim 10 \mathrm{~km})$. Therefore, the hazard for Pointe-à-Pitre is given by a single node. This assessment was carried out using a logic tree approach combined with a Monte-Carlo technique to account for uncertainties.

The second group of parameter uncertainties corresponds to those related to site effects. Two zones of homogeneous amplification were identified in Pointe-à-Pitre. Limited data and information are available so we assume that the amplification factors' values vary around their nominal values by a factor of $10 \%$. When faced with incomplete information, the probability distribution should be selected as the one consistent with the limited knowledge at hand (here satisfying the constraints of lying within an interval), but without introducing unwarranted information. Formally, this can be achieved by choosing the probability distribution with statistical entropy as large as possible, i.e. following the principle of maximum entropy [34]. In our case, the available knowledge is restricted to the bounds of variation, which suggests the use of a uniform probability distribution. The choice of the bounds of this distribution is based on experts' judgements and is expected to have an impact on the results of the sensitivity analysis. Thus, this is further investigated in section 4.3 through a sampling strategy.

In the damage assessment step, parameter uncertainties correspond to the VI assigned to the 15 types of buildings identified in the 80 districts defined during a survey led by BRGM to define a 'Regional Scenario for Seismic Risk' [35]. The main building types are: traditional housing, recent private housing, villa, collective housing and makeshift shelters. Similarly, as for the amplification factors, due to the scarcity of information and data to constrain their uncertainty representations, we adopt the maximum entropy approach [34] and assume that they uniformly vary around their nominal values by a factor of $10 \%$. The influence of the assumed range of variation is further investigated in Section 4.3.

Finally, in the loss assessment step, parameter uncertainties correspond to those related to the average replacement costs associated to each building type. These were estimated by combining, for each class, the construction cost per net floor area and the average area derived 
1 from several French sources: Ministry of the Environment and Housing, National Statistical 2 Agency, Public Reinsurance Institution and so forth (for further details see Réveillère et al. 3 [36]). Based on the available data, the uncertainty in this parameter is represented by a normal 4 probability distribution with a mean corresponding to the evaluated replacement cost and a coefficient of variation of $20 \%$.

[Table 1 about here]

\subsection{Sources of model uncertainty}

Within each phase of the afore-described loss assessment model, different sources of model uncertainty can be identified (Table 1, groups "GMPE", "RAT", and "INV"). These are described here. The influence of the confidence weights assigned by the experts is discussed in Section 4.2.

In the hazard assessment step, we primarily focus on the assumptions for the selection of the GMPEs. Other model-type epistemic uncertainties (e.g., seismo-tectonic zonation and magnitude transformation) are not treated in the present study. Martin et al. [33] showed the negligible influence of two types of seismo-tectonic zonation on the results of regional probabilistic hazard assessment in the case of Guadeloupe; no magnitude transformation was necessary as the earthquake catalogue and the GMPEs were already unified to the same moment magnitude scale. Different combinations for the GMPEs are assumed here (Table 2).

[Table 2 about here]

Each of these combinations is assigned an experts' weight of $25 \%$ so that each combination was considered equally plausible by the experts' panel. Fig. 2 presents the four cumulative probability distributions associated to the rock PGA at Pointe-à-Pitre, resulting from the propagation of the group of uncertain seismic activity parameters for a chosen return period of 475 years (that remains fixed in the present study). 
2 Compilation of the buildings' inventory in each district faces several constraints in practice:

3 financial/time constraints prevents the inspection of each building in detail, because it is often

4 carried out from the roadside; due to the spatial extension of the district, the expert is only able to inspect a few districts, where it is possible to do so; both site surveys and analysis of aerial photos necessarily involves human judgements (in particular during the site surveys) though the recent use of advanced satellite- and ground-based remote sensing techniques have shown promising results [31]. In this context, from one vulnerability inspector to another, the estimation of the ratios of vulnerability classes is expected to vary. This reflects the cumulative contribution of all the different uncertainties within the processing chain. It is chosen to classify such sources of uncertainty as model type. In practice, this should be accounted for by different vulnerability inspectors conducting the same inventory. An example of such an approach is provided by Steimen et al. [37]. Unfortunately, this was not achievable in the present study and we chose to synthetically generate two different inventories by randomly generating a variability of $10 \%$ in the proportions of the original inventory. Both synthetic inventories were assigned a confidence weight of $50 \%$.

At the vulnerability step, we acknowledge the presence of other model-type uncertainties, in particular regarding the choice of the type of vulnerability analysis (here vulnerability is assessed using an intensity-based method by means of the scalar parameter VI). Several authors have recognized the importance of such model-type uncertainty using a OAT-based (parametric) approach. Crowley et al. [38] showed a significant difference in the calculation of seismic risk maps for Italy between two different mechanical-based methods even though these methods share some similarities. Strasser et al. [39] compared different European vulnerability models for a single earthquake scenario close to the city of Istanbul (Turkey). They showed that the predicted distribution of collapsed buildings were similar for the four intensity-based methods, whereas the predictions resulting from the three mechanical/analytical methods were different to a greater degree. Recently, this issue was also investigated by Gehl et al. [40] for a single building using VBSA. They showed that the type of procedure for the generation of fragility curves (whether from dynamic analyses or 30 from a capacity spectrum approach) most influenced the resulting damage assessment 31 compared to other sources of uncertainties (like those associated with the mechanical 32 properties). 
1 A deeper analysis of this issue is worth investigating and constitutes a line of future research,

2 but with the following difficulties to be overcome. Firstly, intensity-based and

3 mechanical/analytical methods have inputs that differ both in types and numbers (in terms

4 ground-motion representation, structural description of the building and so forth). Thus, the number and the nature of the parameter uncertainties differ from one vulnerability method to another, which might pose difficulties to place them at the same "level" when investigating their joint contribution. Secondly, increasing the complexity of vulnerability curves automatically increases the computation time (as pointed out by Crowley et al. [3] in their conclusion), which means conducting the analysis by more advanced procedures such as meta-modelling (see [41] for an example in the field of landslide modelling).

Finally, two different models for repair loss ratios were defined based on Tyagunov et al. [32] and were assigned an equal confidence weight of 50\%. As far as the direct (structural) damage is concerned, the impact of the conversion of damage to economic losses can be large especially when the damage is low [3]. Yet, no matter how accurate the estimates of the conversion parameters values might be, the economic loss assessment still remains incomplete without the inclusion of the effects of intangible and indirect losses. This issue (beyond the scope of the present study) still suffers from numerous gaps, which can be qualified as "scientific uncertainties", either in terms of quantification or in terms of process understanding [42].

[Table 3 about here]

\section{Application}

In this section, we apply the technique described in Section 2.2 to investigate the sensitivity of the loss results to both model and parameter uncertainties (Section 4.1) using the earthquake loss model as described in Section 3. A parametric study is carried out to investigate the influence of experts' assumptions on confidence weights assigned to each model (Section 4.2) and on the coefficients of variation assumed for parameter uncertainty (Section 4.3). It should be underlined that the application to Pointe-à-Pitre has been chosen for demonstration purposes only and all the presented results should not be interpreted as a definitive uncertainty assessment. 


\section{$1 \quad 4.1 \quad$ Models with equal weights}

2 The technique described in Section 2.3 is applied to the loss model described in Section 3.2. 3 The sources of uncertainties are those described in section 3.3 and 3.4; see also Table 1. The 4 output is the total direct economic loss estimated for a return period of 475 years. Samples were generated using the Sobol' quasi-random sequence generator available at http://ipsc.jrc.ec.europa.eu/?id=756. Preliminary convergence tests showed that $N=2000$ yields satisfactory convergence (about $1 \%$ ) for the estimates of the main and total effects. Table 4 shows the main effects calculated for each uncertainty source using the algorithm of Saltelli et al. [20] (routine also available at http://ipsc.jrc.ec.europa.eu/?id=756). In total, $N(n+2)=2000(7+2)=18000$ model evaluations were performed. A single loss evaluation took about 10s so the model evaluations were carried out using grid computing.

\section{[Table 4 about here]}

Analysis of the main effects in Table 4 provides insight into the nature of the considered model. The sum of the seven main effects reaches $96.5 \%$, hence showing that the loss model is additive (see section 2.1) and that the different input parameters interact weakly. This leads to a straightforward quantitative interpretation of the main effects, each of them providing a direct measure for the portion of the sources of input uncertainty on the overall uncertainty in the economic loss estimates, i.e. on its total variance (reaching here 400 million euros). Hence, the analysis can be restricted to the Sobol' indices of first order, i.e. the main effects (and without using the total effects). This only holds for the loss model considered in the present study. In the generic case where the behaviour of the loss model is poorly known (it may be linear, additive or highly non-linear, for example) the difference between the main and the total effects of a given input parameter indicates considerable interactions for that parameter in the model output. This type of analysis is related to the notion of effective dimension [19] and can be of interest when using a loss model in a black-box fashion (see discussion provided by Bommer et al. [4]).

Several observations can be made.

- The most influential source of uncertainty (whether of parameter or model types) corresponds to the seismic activity group (denoted "HAZ") with a main effect of $24 \%$; 
- Model uncertainty on repair loss ratio and on GMPE (denoted "RAT" and "GMPE") are approximately of the same importance (of 19 and 20\%, respectively);

- Uncertainty on the losses (i.e. represented by its variance) is $55 \%$ explained by parameter uncertainty and $41 \%$ by model uncertainty (the remainder is explained by interactions between sources). This means that uncertainty on the losses would be reduced by $41 \%$, if all the models could be unambiguously selected. Similarly, this means that uncertainty on the losses would be reduced by $55 \%$, if all the input parameters could be precisely characterized;

- Model uncertainty on inventories and parameter uncertainty on site effects (denoted "INV" and "LITH") have little influence (2\%). This result should not be considered in an absolute manner, but it reflects the relative contribution of both sources of uncertainty compared to the contributions of the other sources as modelled in the present study. Regarding the former source of uncertainty, this may mostly be explained by the assumption made to represent the uncertainty in the proportions of the original inventory. Regarding the latter source, the reason is more physical and may be associated with the low number of assets in geotechnical zones where site amplification is high;

- Furthermore, the main effects can be analysed by grouping the different sources of uncertainty according to the steps in the loss assessment procedure, namely: hazard assessment (sources of uncertainty "GMPE", "HAZ" and "LITH"), damage assessment ("VI" and "INV"), and economic loss assessment ("COST" and "RAT"). By adding the main effects, the hazard step shows the greatest contribution to the uncertainty in the losses (of almost $45 \%$ ), hence this step should be prioritized in future investigations. The contributions of the damage and loss steps are respectively $37 \%$ and $15 \%$ (the total does not sum to $100 \%$ because of interactions).

These results are discussed in the following section with regards to the experts' assumptions on both confidence weights (here assumed equal) and on the coefficients of variation associated to the sources of parameter uncertainty.

\subsection{Influence of the confidence weights}

To investigate the influence of the confidence weights on the sensitivity results of Figure 3, we adopt a sampling strategy by randomly generating weights following the approach of 
1 Scherbaum et al. [43]. A set of twenty random weights were generated. For each sample of

2 weights, the main effects were re-calculated. Figure 3 depicts the results in the form of

3 boxplots. The horizontal lines correspond to the main effects of the base case with equal

4 experts' weights (Table 4). Note that this type of analysis should not be considered a proper sensitivity analysis (the number of samples being moderate), but rather a parametric one, whose goal is to gain better insight into the impact, on the values of the main effects, of the experts' choices (here the confidence weights and in the following the coefficients of variation, section 4.3) in the mathematical representation of the uncertainty.

The following observations can be made.

- Varying simultaneously the experts' weights of the models not only influences the main effects of the model uncertainty sources, but also those of the parameter uncertainty sources (more specifically the parameter uncertainty "COST", which could increase to the same importance as the uncertainty on hazard "HAZ");

- The choice of the confidence weights mostly influences the main effect associated with the models for conversion of damage to loss "RAT" and with the GMPEs, whereas those associated with the inventory and with site effects are little influenced and remain at levels below 5\%;

- The choice of the confidence weights could greatly decrease the main effect of "RAT" and of GMPEs to less than 10\%. Conversely, the choice of the confidence weights could increase the main effect of GMPEs to the same order as for uncertainty on hazard;.

- The median of the 20 samples of main effects for each uncertainty source does not necessarily correspond to that of the base case assuming equal weights. This indicates that the relationship between the main effects and the experts' weights is complex (and not necessarily linear). 


\section{1}

\subsection{Influence of the coefficients of variation} sources; $17 \%)$ levels below $5 \%$; necessarily linear).

Similarly as for the influence of the confidence weights we generate over the range 10 to $20 \%$, a set of twenty random coefficients of variation (i.e. the size of the parameter uncertainty) associated to the uncertainty sources "LITH", "VI", and "COST". For each coefficient-of-variations sample, the main effects were re-calculated. Figure 4 depicts the results in the form of boxplots. The horizontal lines correspond to the main effects of the base case with equal experts' weights (Table 4). Several observations can be made.

- Varying simultaneously the coefficients of variation not only influences the main effects of the parameter uncertainty sources, but also those of the model uncertainty

- The choice of the coefficient of variation principally influences the value of the main effect associated with the vulnerability assessment "VI" so that it could exceed that associated with the regional hazard assessment as estimated for the base case. This shows that the mathematical representation of the uncertainty in this parameter (here the evaluation of the coefficient of variation) should be carefully constrained and accounted for, because it has the greatest impact on the sensitivity results;

- The choice of the coefficient of variation also influences the main effect associated with the replacement cost so that it could decrease below the one of the base case (of

- The choice of the coefficient of variation had little influence on the main effect associated with the inventory "INV" and with the site effects "LITH", which remain at

- The median of the 20 samples of main effects for each uncertainty source does not necessarily correspond to that of the base case. This indicates that the relationship between the main effects and the size of the parameter uncertainty is complex (and not 
2 In the present article, we described a method to jointly investigate the influence of model and 3 parameter uncertainty on the outcomes of earthquake loss assessment models. The method relying on the variance-based global sensitivity analysis is meant to be generic and can be applied to any kind of model (without any a priori assumption on its mathematical formulation, e.g. linearity or additivity). The procedure is applied to an earthquake loss assessment for Pointe-à-Pitre (Guadeloupe, French Antilles). Four groups of parameter uncertainties are accounted for: parameters of the regional hazard assessment; lithological site effects; vulnerability indices and replacement costs of each building type. Three types of model uncertainty are accounted for: selection of the four ground motion prediction equations, two models to convert damage grades to direct monetary losses and two different inventories of the exposed buildings.

Firstly, the technique can provide a better insight into the loss model behaviour, which can be of interest when using the models in a black-box fashion [4]. In the Pointe-à-Pitre case, the model is found to be principally additive meaning that the different sources of uncertainty weakly interact. Secondly, the technique allows identification of the most important sources of uncertainty. In the considered case, the most influential source of uncertainty (whether of parameter or model type) corresponds to the seismic activity group; uncertainty on losses would be reduced by $41 \%$, if all the models could be unambiguously selected. Thirdly, the technique allows identification of which step of the loss assessment procedure principally drives the overall uncertainty on the output, i.e. which step should be prioritized for further investigations in order to decrease the corresponding uncertainty. In the considered case, the most influential step corresponds to the hazard evaluation grouping the parameter uncertainty of the regional hazard assessment and of the lithological site effects, and the model uncertainty associated with the selection of ground motion prediction equations.

Finally, to test the influence of the experts' assumptions on these results, a sampling strategy, both on the experts' weights on models and on the assumed coefficients of variation of parameter uncertainty, was conducted. It was shown that the choice of the coefficient of variation mostly influences the main effect associated with the vulnerability assessment so that it could exceed that associated with the regional hazard assessment as estimated for the base case. This means that the mathematical representation of this source of uncertainty (e.g., 
1 the most impact on the results of the sensitivity analysis. It is also shown that the choice of the

2 confidence weights could greatly alter the main effect associated with both the models for 3 conversion of damage to loss and with the GMPEs.

4 Though providing useful insights for seismic risk analysis, we acknowledge that the proposed 5 procedure may become computationally expensive, especially when using a loss model with a 6 long run time. A future line of research will, therefore, focus on meta-modelling techniques to 7 overcome such computational difficulties. An example in the field of landslide risk 8 assessment is provided by Rohmer and Foerster [41].

9

\section{Acknowledgements}

11 The research leading to these results has been carried out in the framework of the MATRIX 12 Project, funded by the European Commission's Seventh Framework Program [FP7/200713 2013] under grant agreement $\mathrm{n}^{\circ}$ 265138. We thank the anonymous reviewer for their 14 comments, which led to significant improvements to this article. 


\section{References}

[1] P. Grossi, P. Kleindorfer, H. Kunreuther, The impact of Uncertainty in managing seismic risk: the case of earthquake frequency and structural vulnerability. Technical Report 99-23, The Wharton School, University of Pennsylvania (1999).

[2] S. Steimen, Uncertainties in earthquake Scenarios. PhD Thesis, Swiss Federal Institute of Technology Zürich, 2004.

[3] H. Crowley, J. Bommer, R. Pinho, J. Bird, The impact of epistemic uncertainty on a earthquake loss model. Earthquake Engineering and Structural Dynamics 34 (2005) 16531685.

[4] J.J. Bommer, R. Spence, R. Pinho, Earthquake Loss estimation Models: time to open the Black Boxes, In: Proceedings of the First European Conference on Earthquake Engineering and Seismology, Geneva, Switzerland, 3-8 September, 2006.

[5] J. Rohmer, C. Baudrit, The use of the possibility theory to investigate the epistemic 4 uncertainties within scenario-based earthquake risk assessments, Natural Hazards 56 (2011) 613-632.

[6] N.A. Abrahamson, State of the practice of seismic hazard evaluation, In: Proceedings of GeoEng Conference, Melbourne, Australia, November 19 - 24, 2000.

[7] A. Der Kiureghian, O. Ditlevsen, Aleatory or epistemic? Does it matter?, Structural Safety 31 (2009) 105-112.

[8] B.R. Ellingwood, Kinali K., Quantifying and communicating uncertainty in seismic risk 1 assessment, Structural Safety 31 (2009) 179-187.

2 [9] N. Rabinowitz, D.M. Steinberg, G. Leonard, Logic Trees, Sensitivity Analyses, and Data 3 Reduction in Probabilistic Seismic Hazard Assessment, Earthquake Spectra 14 (1998) 1894 201, doi: http://dx.doi.org/10.1193/1.1585995.

5 [10] S. Molina, C.D. Lindholm, Estimating the confidence of earthquake damage scenarios: 6 examples from a logic tree approach, Journal of Seismology 11 (2007) 299-310, doi: 7 10.1007/s10950-007-9053-3.

8 [11] N. Rabinowitz, D.M. Steinberg, Seismic hazard sensitivity analysis: a multi-parameter 29 approach, Bulletin of the Seismological Society of America 81 (1991) 796-817. 
1 [12] S. Barani, D. Spallarossa, P. Bazzurro, C. Eva, Sensitivity analysis of seismic hazard for

2 Western Liguria (North Western Italy): A first attempt towards the understanding and 3 quantification of hazard uncertainty, Tectonophysics 435 (2007) 13-35.

4 [13] T. Nilsen, T. Aven, Models and model uncertainty in the context of risk analysis,

[14] J.J. Bommer, J. Douglas, F. Scherbaum, F. Cotton, H. Bungum, D. Fäh, , On the Selection of Ground-Motion Prediction Equations for Seismic Hazard Analysis. Seismological Research Letters 81 (2010) 783-793.

[15] A. Saltelli, M. Ratto, T. Andres, F. Campolongo, J. Cariboni, D. Gatelli, M. Saisana, S. Tarantola, Global sensitivity analysis: The Primer, Wiley, Chichester, UK, 304 pp, 2008.

[16] I.M. Sobol', Sensitivity estimates for non linear mathematical models, Mathematical Modelling and Computational Experiments 1 (1993) 407-414.

[17] I.M. Sobol', S.S. Kucherenko, Global sensitivity indices for nonlinear mathematical models, Review of Wilmott Magazine 1 (2005) 56-61.

[18] A. Saltelli, P. Annoni, How to avoid a perfunctory sensitivity analysis, Environmental Modelling and Software 25 (2010) 1508-1517, doi: 10.1016/j.envsoft.2010.04.012.

[19] S. Kucherenko, B. Feil, N. Shah, W. Mauntz, The identification of model effective dimensions using global sensitivity analysis, Reliability Engineering and System Safety 96 (2011) 440-449, doi: 10.1016/j.ress.2010.11.003.

[20] A. Saltelli, P. Annoni, I. Azzini, F. Campolongo, M. Ratto, S. Tarantola, Variance based sensitivity analysis of model output. Design and estimator for the total sensitivity index, Computer Physics Communications 181 (2010) 259-270.

[21] M.J.W. Jansen, Analysis of variance designs for model output, Computer Physics Communications 117 (1999) 35-43.

[22] A. Lopez, S. Stein, T. Dixon, G. Sella, E. Calais, P. Jansma, J. Weber, P. LaFemina, Is there a northern Lesser Antilles forearc block? Geophysical Research Letters 33 (2006), L07313.

[23] F. Beauducel, S. Bazin, M. Bengoubou-Valérius, M.-P. Bouin, A. Bosson, C. AnténorHabazac, V. Clouard, J.-B. de Chabalier, Empirical model for rapid macroseismic intensities 
1 prediction in Guadeloupe and Martinique. Comptes Rendus Geoscience 343 (2011) 717-728,

2 doi:10.1016/j.crte.2011.09.004.

3 [24] O. Sedan, C. Negulescu, M. Terrier, A. Roullé, T. Winter, D. Bertil, Armagedom - A

4 Tool for Seismic Risk Assessment Illustrated with Applications, Journal of Earthquake

$5 \quad$ Engineering 17 (2013) 253-281, doi:10.1080/13632469.2012.726604.

6 [25] B. LeBrun, A.-M. Duval, P.-Y. Bard, O. Monge, M. Bour, S. Vidal, H. Fabriol, Seismic microzoning: a comparison between geotechnical and seismological approaches in Pointe-aPitre (French Western Indies), Bulletin of Earthquake Engineering 2 (2004) 27-50.

[26] A. Roullé, S. Bernardie, Comparison of 1D non-linear simulations to strong-motion observations: A case study in a swampy site of French Antilles (Pointe-à-Pitre, Guadeloupe), Soil Dynamics and Earthquake Engineering 30 (2010) 286-298, doi:

$12 \quad 10.1016 /$ j.soildyn.2009.12.002.

[27] G. Grünthal, European Macroseismic Scale. In: Cahiers du Centre Européen de Géodynamique et de Séismologie, Luxembourg, 15 (1998).

[28] S. Lagomarsino, S. Giovinazzi, Macroseismic and mechanical models for the vulnerability and damage assessment of current buildings, Bulletin of Earthquake Engineering 4 (2006) 445-463.

[29] D.J. Wald, V. Quitoriano, T.H. Heaton, H. Kanamori, C.W. Scrivner, C.B. Worden, TriNet "ShakeMaps": Rapid generation of instrumental ground motion and intensity maps for earthquakes in southern California, Earthquake Spectra 15 (1999) 537-555. [30] G.M. Atkinson, E. Sonley, Empirical relationships between modified Mercalli intensity and response spectra, Bulletin of the Seismological Society of America 90 (2000) 537-544. building inventory for rapid seismic vulnerability assessment: Towards an integrated approach based on multi-source imaging, Soil Dynamics and Earthquake Engineering 36 (2012) 70-83. mapping for Germany, Natural Hazards and Earth System Sciences 6 (2006) 573-586. 
1 [33] C. Martin, P. Combes, R. Secanell, G. Lignon, A. Fioravanti, D. Carbon, O. Monge, B.

2 Grellet, Révision du Zonage Sismique de la France - Etude probabiliste. Technical Report $\mathrm{n}^{\circ}$

3 GTR/MATE/070, 2002.

4 [34] H. Gzyl, The method of maximum entropy. In: F. Bellomo, N. Brezzi (Eds.), Series on 5 Advances in Mathematics for Applied Sciences, 29 (1995), World Scientific Publishing Co, $6160 \mathrm{pp}$.

7 [35] D. Bertil, M. Bengoubou-Valérius, J. Péricat, S. Auclair, Scénarios Départementaux de Risque Sismique en Guadeloupe. Technical Report BRGM/RP-57488-FR (2009) (in French).

[36] A. Réveillère, D. Bertil, J. Douglas, L. Grisanti, S. Lecacheux, D. Monfort, H. Modaressi, H. Müller, J. Rohmer, O. Sedan, Comparative risk assessments for the city of Pointe-à-Pitre (French West Indies): earthquakes and storm surge, In: European Geosciences Union General Assembly, EGU2012-11255, Vienna, Austria, 22-27 April, 2012, Presentation available at: http://presentations.copernicus.org/EGU2012-11255_presentation.pdf.

[37] S. Steimen, D. Fäh, D. Giardini, M. Bertogg, and S. Tschudi. Reliability of Building Inventories in Seismic Prone Regions, Bulletin of Earthquake Engineering 2 (2004) 361 388.

[38] H. Crowley, M. Colombi, B. Borzi, M. Faravelli, M. Onida, M. Lopez, D. Polli, F. Meroni, and R. Pinho. Comparison of seismic risk maps for Italy, Bulletin of Earthquake Engineering 7 (2009) 149-180.

[39] F.O. Strasser, J.J. Bommer, K. Scedilescediletyan, M. Erdik, Z. Ccedilagbrevenan, J. Irizarry, X. Goula, A. Lucantoni, F. Sabetta, I.E. Bal, H. Crowley, C. Lindholm. A Comparative Study of European Earthquake Loss Estimation Tools for a Scenario in Istanbul, Journal of Earthquake Engineering, 12 (2008) 246 - 256.

[40] P. Gehl, T. Ulrich, J. Rohmer, C. Negulescu, A. Ducellier, J. Douglas, Ranking of epistemic uncertainties in scenario-based seismic risk evaluations. In: Proceedings of 11th International Conference on Structural Safety and Reliability, New-York, USA, 16-20 June, 2013, available at: http://hal-brgm.archives-ouvertes.fr/docs/00/82/13/62/PDF/a4sample.pdf

[41] J. Rohmer, E. Foerster, Global sensitivity analysis of large scale landslide numerical models based on the Gaussian Process meta-modelling. Computers and Geosciences 37 (2011) 917-927. 
1 [42] V. Meyer, N. Becker, V. Markantonis, R. Schwarze, J. C. J. M. van den Bergh, L. M.

2 Bouwer, P. Bubeck, P. Ciavola, E. Genovese, C. Green, S. Hallegatte, H. Kreibich, Q.

3 Lequeux, I. Logar, E. Papyrakis, C. Pfurtscheller, J. Poussin, V. Przyluski, A. H. Thieken, C.

4 Viavattene. Review article: Assessing the costs of natural hazards - state of the art and

5 knowledge gaps, Natural Hazards and Earth System Sciences, 13 (2013) 1351-1373,

6 doi:10.5194/nhess-13-1351-2013.

7 [43] F. Scherbaum, J.J. Bommer, H. Bungum, F. Cotton, N.A. Abrahamson, Composite 8 ground-motion models and logic trees: methodology, sensitivities and uncertainties, Bulletin

9 of the Seismological Society of America 95 (2005) 1575-1593, doi: 10.1785/0120040229.

10 [44] T. Takahashi, S. Kobayashi, Y. Fukushima, J.X. Zhao, H. Nakamura, P.G. Somerville, A 11 spectral attenuation model for Japan using strong motion data base. In: Proceedings of the 12 Sixth International Conference on Seismic Zonation, Palm Springs, California, 12-15 13 November, 2000.

14 [45] C. Berge-Thierry, F. Cotton, O. Scotti, D. Griot-Pommera, Y. Fukushima, New empirical 15 response spectral attenuation laws for moderate European earthquakes. Journal of Earthquake 16 Engineering 7 (2003) 193-222.

17 [46] R.R. Youngs, S.-J. Chiou, W.J. Silva, J.R. Humphrey, Strong ground motion attenuation 18 relationships for subduction zone earthquakes, Seismological Research Letters 68 (1997) 19 94-127. 
2 Table 1. Description of the sources of uncertainty and assumptions for representing them in

3 the Pointe-à-Pitre application.

\begin{tabular}{|c|c|c|c|}
\hline $\begin{array}{c}\text { Uncertainty } \\
\text { source }\end{array}$ & Type & Description & Representation \\
\hline “HAZ” & Parameter & $\begin{array}{l}\text { Seismic activity } \\
\text { parameters of the } \\
\text { PHSA }\end{array}$ & Gaussian (normal) distribution \\
\hline “LITH” & Parameter & $\begin{array}{l}\text { Amplification factor of } \\
\text { lithological site effect }\end{array}$ & $\begin{array}{l}\text { Uniform distribution with variation } \\
\text { of } 10 \% \text { from the estimated values }\end{array}$ \\
\hline "VI" & Parameter & Vulnerability index & $\begin{array}{l}\text { Uniform distribution with variation } \\
\text { of } 10 \% \text { from the estimated values }\end{array}$ \\
\hline "COST" & Parameter & $\begin{array}{l}\text { Average cost of each } \\
\text { type of building }\end{array}$ & $\begin{array}{l}\text { Gaussian (normal) distribution with } \\
\text { variation of } 20 \% \text { from the estimated } \\
\text { values }\end{array}$ \\
\hline "GMPE" & Model & $\begin{array}{l}\text { Selection of the } \\
\text { GMPE, see Table } 2\end{array}$ & $\begin{array}{l}\text { Discrete variable with values } \\
\text { uniformly taken from }\{1,2,3,4\} \text { for } \\
\text { each GMPE }\end{array}$ \\
\hline "RAT" & Model & $\begin{array}{l}\text { Conversion of repair } \\
\text { loss ratio, see Table } 3\end{array}$ & $\begin{array}{l}\text { Discrete variable with values } \\
\text { uniformly taken from }\{1,2\} \text { for } \\
\text { each model of conversion }\end{array}$ \\
\hline "INV" & Model & $\begin{array}{l}\text { Inventory: assessment } \\
\text { of the ratio of } \\
\text { vulnerability } \\
\text { typologies within each } \\
\text { city district }\end{array}$ & $\begin{array}{l}\text { Discrete variable with values taken } \\
\text { from }\{1,2\} \text { for each inventory }\end{array}$ \\
\hline
\end{tabular}

5 Table 2. Combinations of GMPE models assumed for the application of Pointe-à-Pitre

\begin{tabular}{lll}
\hline \multicolumn{1}{c}{ Combination } & \multicolumn{1}{c}{ Shallow crustal events } & \multicolumn{1}{c}{ Subduction events } \\
\hline TT & Takahashi et al. [44] & Takahashi et al. [44] \\
BT & Berge-Thierry et al. [45] & Takahashi et al. [44] \\
BY & Berge-Thierry et al. [45] & Youngs et al. [46] \\
TY & Takahashi et al. [44] & Youngs et al. [46] \\
\hline
\end{tabular}


1 Table 3. The two conversion models of repair loss ratio (adapted from Tyagunov et al. [30])

2 considered here

\begin{tabular}{llllll}
\hline \multicolumn{1}{c}{ Damage grade } & D1 & D2 & D3 & D4 & D5 \\
\hline Repair loss ratio \% (conversion model 1) & 0 & 10 & 40 & 80 & 100 \\
Repair loss \% (conversion model 2) & 1 & 20 & 60 & 100 & 100 \\
\hline
\end{tabular}

3

4 Table 4. Main effects (Sobol' indices of first order) associated with the four uncertainty 5 sources of type "parameter" ("HAZ", "LITH", "VI" and "COST") and the three ones of type 6 "model" for the case of equal experts" confidence weights ("GMPE", "RAT" and "INV"). See 7 Table 1 for further description of the uncertainty sources.

\begin{tabular}{cccccccc}
\hline Uncertainty Source & HAZ & LITH & VI & COST & GMPE & RAT & INV \\
\hline Main effect $(\%)$ & 24 & 1.5 & 13 & 17 & 19 & 20 & 2 \\
\hline
\end{tabular}

8

9 
A)

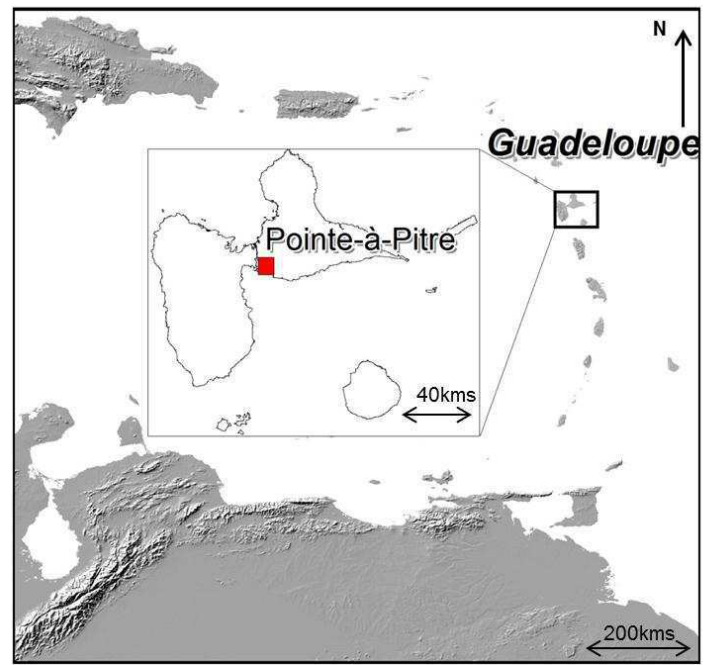

B)

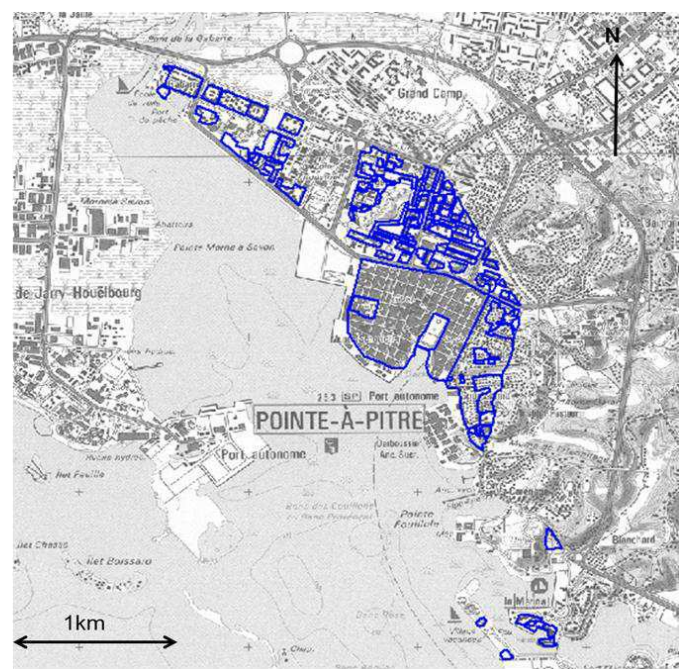

3 Figure 1. A) Location of the case study, Pointe-à-Pitre (Guadeloupe, France); B) Zonation of the Pointe-à-Pitre urbanized area into 80 districts for vulnerability assessment

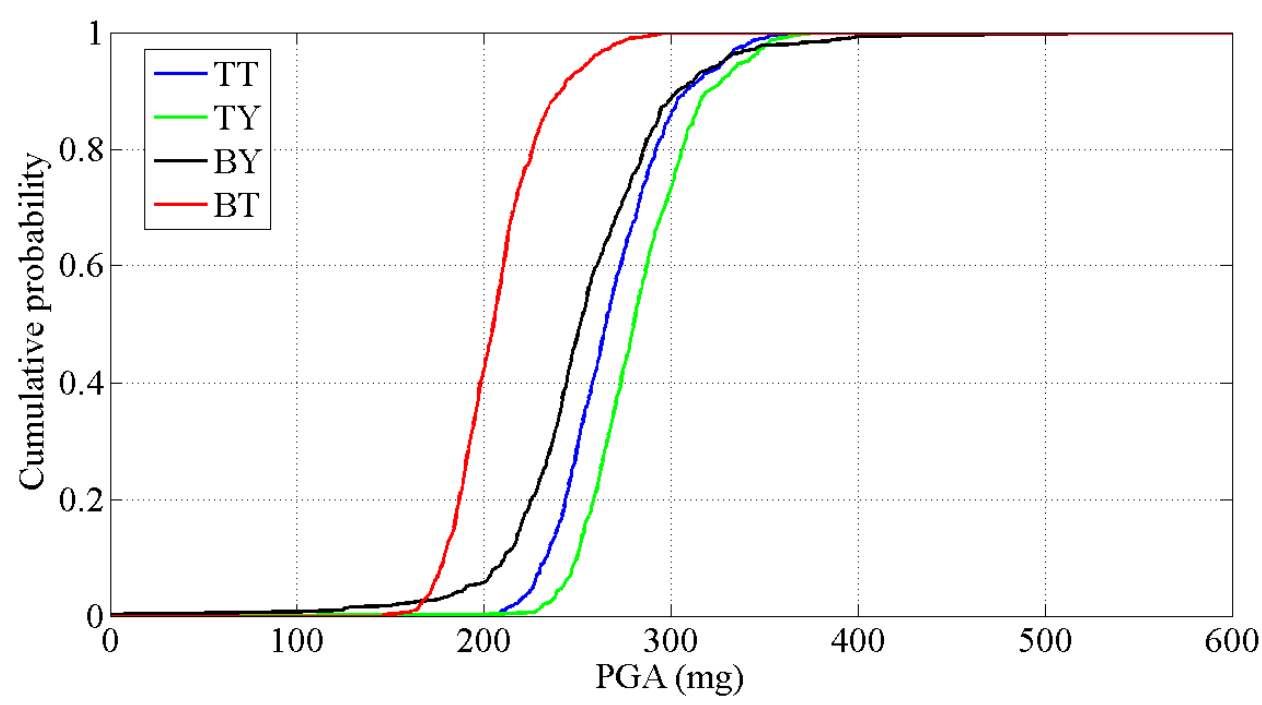

7 Figure 2. Cumulative probability distribution for the PGA on rock at Pointe-à-Pitre for a return period of 475 years. Each curve corresponds to a combination of GMPEs. TT:

9 Takahashi et al. [44] for both shallow crustal and subduction events; BT: Berge-Thierry et al. [45] for shallow crustal earthquakes and Takahashi et al. [44] for subduction events; BY:

11 Berge-Thierry et al. [45] for shallow crustal earthquakes and Youngs et al. [46] for subduction events; and TY: Takahashi et al. [44] for shallow crustal earthquakes and Youngs et al. [46] for subduction events. 


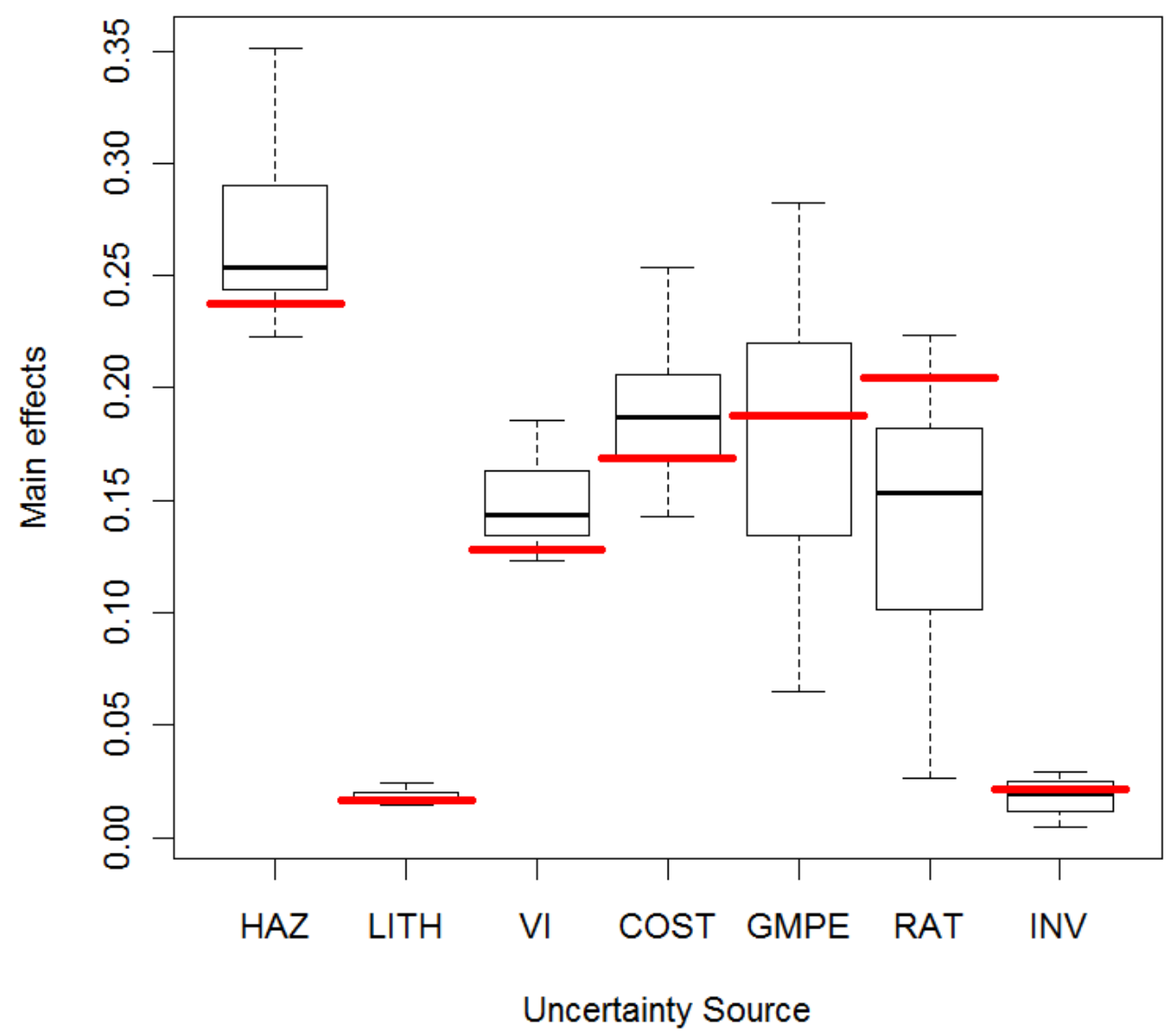

1

2 Figure 3. Boxplot summarizing the influence on the sensitivity results of twenty randomly 3 chosen confidence weights on the models. Bottom and top of the box are the 25th and 75th 4 percentile, the band near the middle of the box is the median, and the ends of the whiskers 5 represent the minimum and maximum of the sample. The red horizontal lines are the values of 6 the main effects for the base case with equal weights (Table 4). 


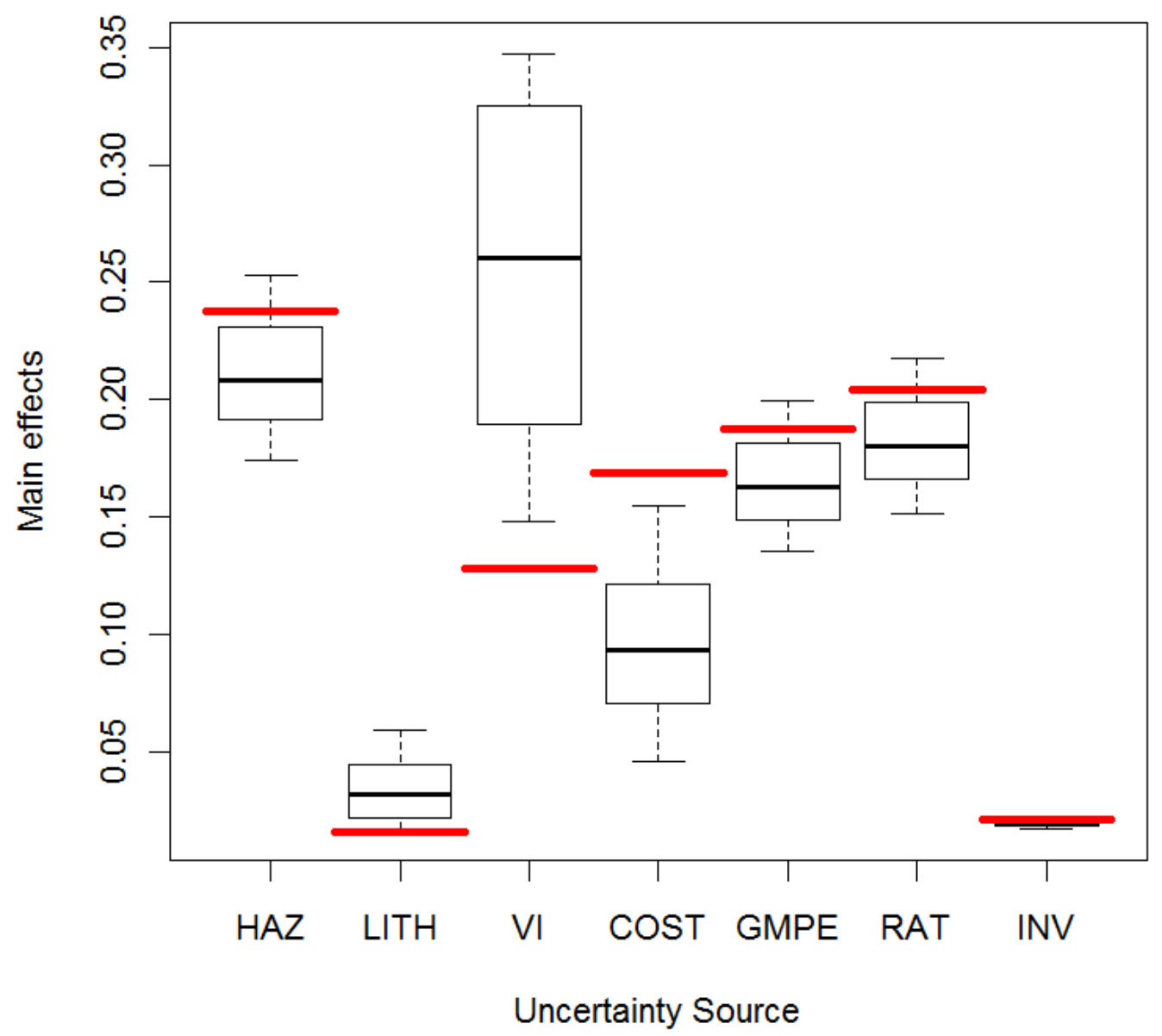

1

2 Figure 4. Boxplot summarizing the influence on the sensitivity results of twenty randomly 3 chosen coefficients of variation of uncertainty sources "LITH", "VI" and "COST". Bottom 4 and top of the box are the 25th and 75th percentile, the band near the middle of the box is the 5 median, and the ends of the whiskers represent the minimum and maximum of the sample.

6 The red horizontal lines are the values of the main effects for the base case with equal weights 7 (Table 4). 